\title{
Conexão entre os Sistemas de Referência Óptico e Rádio
}

\author{
Connection between Optical and Radio Reference Systems
}

\author{
Alan Alves-Brito* \\ Instituto de Astronomia, \\ Geofísica e Ciências Atmosféricas - USP \\ São Paulo - SP - Brasil - 11000-000 \\ V.A.F. Martin ${ }^{\dagger}$ and P.C.R. Poppe ${ }^{\ddagger}$ \\ Laboratório Nacional de Astrofísica - MCT \\ Observatório Astronômico Antares \\ Departamento de Física - UEFS \\ Campus Universitário, Km 03, BR 116 \\ Feira de Santana - BA - Brasil - 44031-460
}

\begin{abstract}
Astrometria é a parte da Astronomia dedicada à medida de distâncias, movimentos, dimensões, posições e geometria dos corpos celestes, definida dentro da estrutura da Mecânica Newtoniana. Muitos instrumentos astrométricos têm sido utilizados em programas ou propostas específicos. Neste trabalho discutimos as limitações da Astrometria baseada na Terra e suas contribuições ao estabelecimento de um referencial absoluto usando o Astrolábio Foto-elétrico (ASPHO). Os principais resultados obtidos para a posição de rádio-estrelas são mostrados e discutidos confimando a oportunidade de programas de astrolábio primático dedicados à conexão de referenciais ópticos e de rádio. Apresentamos a medida dos ângulos de rotação entre o sistema de rádio e nossos dados ópticos bem como uma comparação com as posições óptico-rádio obtidas por outras técnicas.
\end{abstract}

Palavras-chave: Astrometria, Astrolábio Fotoelétrico, Referencial Óptico, Referencial de Rádio.

Astrometry is the part of Astronomy devoted to the measurement of the distances, motions, dimensions, positions and geometry of celestial bodies, defined in such a way that Newtonian mechanics is valid. Several astrometric instruments have been using for various particular programs or purposes. In this work we discuss the limitations of the ground based astrometry and its contributions to the establishment of an absolute reference frame by using the Photoelectric Astrolabe (ASPHO). The main results obtained for the position of radio stars are shown and discussed confirming the opportunity of prismatic astrolabe programs devoted to the connection of the optical and radio reference frames. The measurement of the rotation angles between the radio system and our optical data as well as a comparison with optical-radio positions obtained by other techniques are presented.

Keywords: Astrometry, Photoelectric Astrolabe, Optical Reference, Radio Reference.

\section{ASTROMETRIA - FAZENDO O CENSO DO UNIVERSO}

\footnotetext{
*Endereço Eletrônico: abrito@astro.iag.usp.br

${ }^{\dagger}$ Endereço Eletrônico: vmartin@uefs . br

${ }^{\ddagger}$ Endereço Eletrônico: poppe@uefs.br
}

A Astrometria caracteriza-se como o mais antigo dos diversificados ramos da Astronomia - Astrofísica, Cosmologia, Planetologia, Ensino e outros - tendo como principal finalidade medir distâncias, movimentos, posições, dimensões e geometria dos corpos celestes; estudar a dinâmica do Sistema Solar, estrelas e sistemas estelares, estabelecendo para tanto, 
sistemas inerciais de referência. Um trabalho meticuloso de censo do Universo observado. A Astrometria constitui-se na maior técnica astronômica de medida de parâmetros essenciais para a descrição dinâmica do Universo [1-6].

Tendo em vista que todos os corpos celestes movimentam-se (o Universo é dinâmico) o estudo desses movimentos torna-se necessário à medida que buscamos compreender as diversas estruturas no Universo, procurando determinar a posição desses objetos e as variações temporais dessas posições com grande precisão [7]. Podemos ainda destacar que foram observações e medidas sistemáticas das posições dos corpos celestes (Astrometria) que introduziram o moderno conceito de método científico que gerencia a produção intelectual ocidental nas várias áreas do conhecimento.

Classicamente, a Astrometria cobre o estudo do movimento da Terra. São sempre feitas observações de solo de forma a se criar um conjunto de dados. A cada nova observação são feitas referências às anteriores e aos movimentos orbital e rotacional da Terra. Separar estas duas componentes do movimento da Terra seria a maior dificuldade enfrentada pela Astrometria. Dentro desse contexto, os erros sistemáticos que surgem da rotação da Terra, materializados na precessão, introduzem ao sistema de movimento próprio das estrelas uma polarização sistemática que afetará a determinação do movimento dos grupos estelares bem como da rotação galáctica. Esta é a razão principal pela qual a Astrometria busca estabelecer um sistema de referência o mais inercial possível, um sistema inercial de coordenadas no qual todos os efeitos terrestres sejam desprezíveis e sem nenhuma rotação. Este é o tão sonhado sistema absoluto ou inercial de referência [1].

Em suma, cabe à Astrometria às seguintes atribuições:

- estabelecer um sistema de referência local o mais inercial possível;

- determinar o movimento próprio das estrelas com o objetivo de investigar a cinemática estelar;
- estabelecer uma identificação única dos objetos celestes observados em diferentes comprimentos de onda, para interpretação de estruturas;

- estudar o movimento dos objetos extragalácticos para avanço nas pesquisas cosmológicas;

- materializar um sistema inercial global de referência;

- estimar constantes astronômicas fundamentais e a idade do Universo.

\section{A. Sistemas Inerciais de Referência}

As teorias dinâmicas implicam na existência de sistemas de referência descritos no espaço e no tempo e quando aplicadas a sistemas físicos (ditos sistemas reais), materializam as escalas de tempo e de espaço. O problema básico da Astrometria seria, respaudada nas equações newtonianas, definir um robusto eixo de coordenadas, fixo em direção, ao qual poderíamos referir posições de estrelas e objetos do sistema solar. A estreita relação entre um sistema inercial de referência e as leis de Newton fica mais clara quando recorremos ao conceito de inércia como uma propriedade característica de tal sistema. Esta relação parece nos levar a um conceito dinâmico do sistema inercial de referência, quando na verdade, apresentamos uma definição cinemática, e isso é levado em conta nas teorias da Relatividade Especial e Geral de Einstein. Em se tratando da Teoria Geral da Relatividade o sistema de coordenadas encontra-se centrado no baricentro do sistema solar e as coordenadas quadridimensionais são escolhidas de tal forma que a métrica do sistema obedeça a relação:

$$
\mathrm{ds}^{2}=\mathrm{c}^{2} \mathrm{~d} \tau^{2}=\mathrm{c}^{2} \mathrm{dt}^{2}-\mathrm{dx}^{2},
$$

onde $c$ é a velocidade da luz, $t$ e $\tau$ são, respectivamente, o tempo medido na coordenada e o tempo próprio, e $x=\left(c t, x_{1}, x_{2}, x_{3}\right)$ são as quatro coordenadas espaço-temporais [2, 3]. Vale 
ressaltar que os efeitos da Relatividade Geral sobre objetos do sistema solar são da ordem de $(v / c)^{2}$, onde $\mathrm{v}$ é a velocidade típica destes objetos. As equações do movimento, expressas com base na métrica espaço-temporal, diferem na ordem de $10^{8}$ das equações escritas no formalismo Newtoniano. Estas diferenças podem ser consideradas como pequenas correções na formulação clássica newtoniana. Dentro desse contexto, um sistema inercial para astrometria seria um sistema desprovido de qualquer aceleração ou forças como a centrífuga e a de Coriolis. Isso nos conduz a um sistema inercial baseado na Lei da Gravitação Universal de Newton e na interação dos corpos.

Precisamos agora definir para a Astrometria planos e direções sobre os quais podemos expressar sistemas inercias de coordenadas, descritos dinamicamente. Segundo o Princípio de Mach, um sistema inercial de referência é estabelecido quando o sistema de coordenadas que o caracteriza mantém-se sem efeitos de rotação ou totalmente em repouso em relação ao Universo, onde a idéia de inércia surge como conseqüência da densidade de matéria do Universo. O Princípio de Mach traz para a Astrometria um sistema inercial de referência que pode ser estabelecido pela análise da posição de objetos extragalácticos, que por estarem tão distantes podem ser considerados em estado de repouso. Isso não está explícito nos textos que tratam da problemática da definição de um sistema inercial de referência para a Astrometria [2].

Como vimos, o tempo também é um parâmetro importante na descrição dinâmica de um corpo. Em Astrometria temos o Tempo Universal (rotação da Terra), Tempo das Efemérides (Movimento Orbital da Terra) e o Tempo Atômico Internacional (Transição Atômica Hiperfina). No que diz respeito às coordenadas espaciais, a Astrometria utiliza catálogos fundamentais como base de referência na tentativa de estabelecer um sistema inercial.

Há uma série de recomendações ou critérios quanto à definição de um sistema de referência perfeito em Astronomia de Posição:

- O primeiro critério é de observabili- dade: os sistemas de referência devem ser bastante próximos de informações observacionais. Alguns cuidados são necessários no momento de definir uma base de referência. Por exemplo, nunca poderíamos definir uma base de referência terrestre baseada no vetor momento angular total. Fisicamente, esta restrição está associada a atuação de torques sobre os planos terrestres principais, que são utilizados na definição da base de referência.

- O segundo critério é o da simplicidade: sabemos que quanto mais simples uma teoria científica e quanto maior seu poder de previsibilidade (previsibilidade aqui não no sentido de um determinismo stricto sensu), mais poderosa ela é. Assim também devem ser os sistemas de referência. Conceitualmente, o mais simples possível.

- O terceiro critério é o de estabilidade: a Astronomia trabalha com uma base de tempo que varia de uma escala que vai dos infinitésimos aos infinitos. Dessa forma, precisamos ter cuidado quando passamos de um sistema de referência a outro, pois nessa mudança podem ocorrer incrementos de ruídos e erros computacionais.

- Um quarto critério é o de unicidade: todos os elementos do sistema de referência devem ser univocamente identificados. Um exemplo disso é o fato de que nem sempre uma efeméride e uma integração numérica fornecem os mesmos valores para determinado fenômeno.

- Um quinto critério é o de densidade: quanto maior o número de objetos usados para definir o sistema, mais consistente ele será.

A Tabela I resume os principais tipos de catálogos em Astrometria. 
TABELA I: Principais sistemas de referência em Astrometria

\begin{tabular}{lcccccc}
\hline \hline Nome & Objeto & $\#$ & Magnitude & Época & MODO & Status \\
\hline FK5 & Estrelas & 1535 & $<7$ & 1945 & Astrometria Óptica & Cat. Fundamental \\
FK5-sup & Estrelas & 3117 & $<9.50$ & 1945 & Astrometria Óptica & Cat. Fundamental \\
ICRF & Fontes Rádio & 212 & - & 1987 & VLBI & SRI a partir de 1998 \\
ICRF & Fontes Extragal. 398 (Ref.) & - & 1987 & VLBI & SRI a partir de 1998 \\
HIPPARCOS & Estrelas & 117950 & $<12$ & 1991,25 & Satélite & ICRF no óptico \\
\hline
\end{tabular}

\section{ASTROLÁBIO FOTOELÉTRICO}

O astrolábio foi um instrumento inventado por Hiparcos, com a finalidade de medir a altura de um astro acima do horizonte. Em meados do século XX, com a finalidade de estudar o movimento do pólo e monitorar a variação da velocidade de rotação da Terra, através da observação visual de estrelas com magnitude inferior a 6 , foi construído o astrolábio à prisma de Danjon. Vale ressaltar que a terminologia astrolábio só foi mantida por razões históricas. O astrolábio Danjon, bem como o fotoelétrico nada têm a ver com o astrolábio inventado por Hiparcos.

A Astrometria conta com alguns grandes catálogos que concretizam um sistema de referência: o Catálogo Hipparcos (alta precisão, mas passível à degradação devido ao movimento próprio das estrelas), o $F K 5$, o $F K 6$ e os sistemas de referência extragalácticos quaseinercias em comprimentos de onda rádio. A grande contribuição científica do astrolábio é a interligação entre todos esses sistemas de referência, a fim de torná-los acessíveis às janelas astrométricas e por isso os astrômetras procuraram substituir a aquisição de dados mecânico-visual do astrolábio Danjon por um sistema automático e de fotossensores no Astrolábio Fotoelétrico, para aumentar o desempenho observacional do referido instrumento [8-10].

O princípio de funcionamento do astrolábio implica uma visualização bidimensional e uma resolução temporal apurada. Ao contrário do instrumento óptico à prisma de Danjon, o Astrolábio Fotoelétrico colhe informações observacionais através de uma fotomultiplicadora e, por isso, requer uma exigência no nivelamento e em sua robustez. Há outros pequenos problemas que passam pela eficiência quântica, amplificação, o que limita em aproximadamente 8 a magnitude do instrumento. $\mathrm{O}$ princípio físico associado à fotomultiplicadora é o Efeito Fotoelétrico. A recepção do sinal é por contagem de fótons e por esse motivo deve-se tomar cuidado com o nivelamento (garantido pelo banho de mercúrio), pois a intensidade do sinal é diretamente proporcional ao número de fótons detectados na coletora.

O procedimento básico do instrumento é obter duas imagens de um mesmo objeto celeste com o auxílio de um esquadro óptico (o esquadro óptico define o lugar geométrico de observação), formadas a partir de dois espelhos e por uma superfície refletora de mercúrio que estabelece o horizonte artificial do local. No momento em que duas imagens se cruzam, o objeto observado encontra-se a uma distância zenital igual ao ângulo definido pelo esquadro óptico. Este instrumento fornece informações bastante precisas sobre a posição do objeto observado no céu, com a ajuda de um prisma à transmissão para observações a $30^{\circ}$ e $45^{\circ}$ de distância zenital (ver detalhes em [11] \& [12]).

A aquisição dos dados é feita com a ajuda de um sistema eletrônico. As imagens são moduladas por uma grade antes de chegarem na superfície sensível do fotomultiplicador. Acoplado ao instrumento também funciona, na coleta e análise de dados, uma estação de pro- 
cessamento e outros periféricos.

\section{MONTAGEM DO PROGRAMA OBSERVACIONAL}

A missão astrométrica espacial HIPPARCOS, reconhecidamente, caracterizou-se como um dos marcos na Astrometria. Apesar dos problemas técnicos decorrentes do lançamento, o satélite astrométrico forneceu bons resultados. Conhece-se a posição e a paralaxe de, aproximadamente, 120.000 estrelas dentro da precisão de 0,7 mas (corresponde ao ângulo no qual vemos uma ervilha a mil quilômetros de distância) com limite superior, além dos movimentos próprios, cuja precisão é limitada a 1 mas/ano, devido à curta duração da missão $[12,13]$.

Em 1998, buscamos definir o programa observacional do Astrolábio Fotoelétrico (ASPHO) no sítio observacional de Feira de Santana: a formação do conjunto de estrelas observáveis com o ASPHO no sítio do Observatório Astronômico Antares - OAA $(\phi=$ $-12^{\circ} 15^{\prime}$ latitude geográfica local; $\lambda=38^{\circ} 57^{\prime} \mathrm{W}$ longitude geográfica local). Utilizamos as coordenadas de estrelas pertencentes ao catálogo HIPPARCOS por este se constituir como o mais inercial dos catálogos astrométricos. Foram selecionadas, aproximadamente, 18500 estrelas à $30^{\circ}$ e 27200 estrelas à $45^{\circ}$ de distância zenital.

Em seguida, dispondo do catálogo HIPPARCOS, desenvolvemos um programa computacional codificado na linguagem Fortran para separar, das aproximadamente 120000 estrelas do catálogo, apenas aquelas que poderiam ser observadas no OAA. Além disso, tivemos a preocupação de incluir o maior número de estrelas radiofontes aos nossos grupos estelares, pois estas são os melhores objetos disponíveis para observações com o astrolábio capazes de fazerem a conexão entre os sistemas de referência óptico e rádio e vice-versa.

As estrelas de dupla passagem também entraram em nossos grupos. De posse destas estrelas e por intermédio de um outro programa em Fortran, calculamos o Tempo Sideral (TS) e o Azimute $(\mathrm{Az})$ das estrelas a partir da ascensão reta $(\alpha)$ e da declinação $(\delta)$ das mesmas. Em seguida, separamos o conjunto de estrelas em intervalos de magnitude. Analisamos os resíduos brutos obtidos de aproximadamente 2 anos de observações a $30^{\circ}$ e a $45^{\circ}$ de distância zenital do conjunto de estrelas do programa observacional do Astrolábio Fotoelétrico, sítio Observatoire de la Côte d'Azur - OCA, França $\left(\phi=00 h 27 m 42 s, 44 E ; \lambda=+43^{\circ} 44^{\prime} 55 ", 011 ;\right.$ $h=1260 \mathrm{~m})$, totalizando 59719 resíduos, sendo que 34031 são a $30^{\circ}$ e 25688 a $45^{\circ}$ ( 59000 observações) de distância zenital.

Utilizamos diversos programas codificados em linguagem de programação científica Fortran para o cálculo dos resíduos médios e a média dos resíduos médios a Este e a Oeste das estrelas do programa observacional. O mesmo procedimento foi realizado para a escolha das estrelas radiofontes e das estrelas em condição de máxima digressão (estrelas cujo cosseno do ângulo paralático é nulo), tanto a $30^{\circ}$ quanto a $45^{\circ}$ de distância zenital.

\section{A. Conexão óptico e Rádio}

Tendo em vista a concepção da Esfera Celeste (ver todos os detalhes em [14]), é conveniente, por razões de simetria, que usemos um sistema de coordenadas esféricas para definir a posição de qualquer ponto (astro) na superfície da esfera. Temos então um vetor $\vec{r}=\vec{r}(\theta, \phi)$ para as coordenadas esféricas. Como a coordenada $r$ é constante, podemos considerála, arbitrariamente, igual a unidade, definindo a direção do astro em relação ao observador através do ângulo zenital $\theta$ e do ângulo azimutal $\phi$.

Podemos associar a todo sistema de referência um trio de direções ortogonais no espaço que constituem os eixos dos sistemas. Em princípio, a escolha dessas direções é arbitrária e, levando-se em conta a grande estabilidade das posições das radiofontes extragalácticas, podemos afirmar que estas direções são fixas no espaço, mas são diferentes de um 
sistema para outro [14].

A orientação relativa entre dois sistemas $L$ e $S$, tendo-se o mesmo centro (não há translação), é identificada a partir de três rotações diretas cujos ângulos são denomina$\operatorname{dos} A_{1}, A_{2}, A_{3}$, em torno dos eixos $x, y, z$, respectivamente.

A transformação de coordenadas de um ponto na direção $V 1$, no sistema $L$, e $V 2$, no sistema $S$, efetua-se através da relação:

$$
\mathrm{V} 1=[\mathrm{M}] \mathrm{V} 2
$$

Aqui $M$ é a matriz de rotação entre os eixos dos dois sistemas de referência. Ela é resultado das rotações em torno dos eixos $x, y$ e $z$ representadas por $M_{x}, M_{y}$ e $M_{z}$ :

$$
M=M_{x} M_{y} M_{z}
$$

onde:

$$
\begin{aligned}
& \mathbf{M}_{\mathbf{x}}=\left(\begin{array}{ccc}
1 & 0 & 0 \\
0 & \cos \mathrm{A}_{1} & \sin \mathrm{A}_{1} \\
0 & -\sin \mathrm{A}_{1} & \cos \mathrm{A}_{1}
\end{array}\right) \\
& \mathbf{M}_{\mathbf{y}}=\left(\begin{array}{ccc}
\cos \mathrm{A}_{2} & 0 & -\sin \mathrm{A}_{2} \\
0 & 1 & 0 \\
\sin \mathrm{A}_{2} & 0 & \cos \mathrm{A}_{1}
\end{array}\right) \\
& \mathbf{M}_{\mathbf{z}}=\left(\begin{array}{ccc}
\cos \mathrm{A}_{3} & \sin \mathrm{A}_{3} & 0 \\
-\sin \mathrm{A}_{3} & \cos \mathrm{A}_{3} & 0 \\
\sin \mathrm{A}_{2} & 0 & 1
\end{array}\right)
\end{aligned}
$$

Como $A_{1}, A_{2}, A_{3}$ são ângulos de milésimos de segundo de grau (infinitesimais em radianos), podemos utilizar série de potências de Taylor para pequenos ângulos e negligenciar os termos de segunda ordem e ordens superiores. Daí:

$$
\mathbf{M}=\left(\begin{array}{ccc}
1 & \mathrm{~A}_{3} & -\mathrm{A}_{2} \\
-\mathrm{A}_{3} & 1 & 0 \\
\mathrm{~A}_{2} & -\mathrm{A}_{1} & 1
\end{array}\right)
$$

A avaliação da orientação relativa entre sistemas se faz sobre a base de fontes comuns. A mesma fonte tem coordenadas diferentes em diferentes sistemas, já que seus eixos não estão sobre a mesma direção.

Após algumas manipulações matemáticas, as posições ópticas das fontes podem ser postas em função dos ângulos de rotação pelas equações abaixo:

$$
\begin{aligned}
\Delta \alpha= & A_{1} \sin \delta_{2} \cos \alpha_{2} \sec \delta_{1} \\
& +A_{2} \sin \delta_{2} \sin \alpha_{2} \sec \delta_{1} \\
& -A_{3} \cos \delta_{2} \sec \delta_{1} \\
\Delta \delta=- & \mathrm{A}_{1} \sin \alpha_{2}+\mathrm{A}_{2} \cos \alpha_{2}
\end{aligned}
$$

\section{RESULTADOS}

A turbulência atmosférica, a refração, os movimentos da Terra e a impossibilidade de se observar o céu em sua totalidade, representam as principais limitações observacionais da astrometria de solo. A refração para o instante da observação, é geralmente corrigida a partir de valores interpolados das temperaturas do ar (seco e úmido) e da pressão atmosférica, tomados antes e após cada conjunto de estrelas observadas. Entretanto, é bem conhecido que alguns dos métodos utilizados para o cálculo da refração não são totalmente satisfatórios, subsistindo consideráveis anomalias (aleatórias e sistemáticas) para os diferentes perfis atmosféricos. Qualquer que seja o detetor utilizado, a atmosfera terrestre representa a maior fonte de dispersão para as medidas realizadas com os mais variados instrumentos de solo [15].

Um dos requisitos mais importantes para se trabalhar com a astrometria estelar de solo, 
com qualquer tipo de instrumento, é definir a magnitude das estrelas a serem observadas. Para o caso do ASPHO, o limite de magnitude gira em torno de 8 . O segundo requisito é saber qual a faixa de declinação (campo de estrelas) é possível de ser observada no sítio desejado com o instrumento desejado. O terceiro requisito é a distribuição em azimute que deve ser homogênea para o caso de trabalho com astrolábios.

Já sabemos que para obtermos as posições de corpos materiais com a finalidade de estudar seus movimentos, é aconselhável estabelecer-se um sistema de referência inercial. Devido a alta precisão com que se pode medir suas posições, as observações de estrelas têm o propósito de materializar estes sistemas.

As posições e movimentos próprios precisos de rádio-estrelas com contrapartida óptica são necessários para a determinação direta da conexão entre o sistema de referência rádio VLBI (Very Long Baseline Interferometry) e o sistema de referência óptico, de solo, baseado em estrelas fundamentais.

Na prática, a dificuldade para a conexão reside no fato dos objetos usados na materiali- zação do sistema VLBI (quasares) possuírem magnitude na ordem de 15 chegando a 17 e 18 , enquanto as estrelas de catálogos oriundos de observação de solo com o ASPHO alcançam magnitude máxima de 8.

Podemos definir o modelo astrométrico admitindo-se que, em primeira aproximação, as estrelas apresentam um movimento retilíneo uniforme relativo ao baricentro do sistema solar. Fisicamente, corresponde à uma aceleração nula para essas estrelas e o fortalecimento para a idéia de um sistema inercial de referência.

A partir das fontes rádio, somos capazes de determinar efeitos sistemáticos locais entre o sistema rádio e o sistema óptico dentro do modelo de um sistema inercial de referência. Consideremos que $\Delta \alpha$ e $\Delta \delta$ (dadas na secção 3) sejam as diferenças entre as posições dos catálogos nos dois sistemas, onde as incógnitas $A_{1}, A_{2}$ e $A_{3}$ (ângulos de rotação) são determinadas utilizando-se o método de Gram-Smith modificado para inversão de matrizes (MGS).

A Tabela II mostra os ângulos de rotação dos eixos $x, y$ e $z$, com as respectivas dispersões. O nosso sistema é apresentado e é feita uma comparação com outros sistemas $[6,16]$.

TABELA II: Ângulos de rotação de diversos catálogos

\begin{tabular}{lcccc}
\hline \hline Catálogo & $\mathrm{A}_{1} \pm \sigma[\mathrm{mas}]$ & $\mathrm{A}_{2} \pm \sigma[\mathrm{mas}]$ & $\mathrm{A}_{3} \pm \sigma[\mathrm{mas}]$ & $\sigma_{\text {geral }}[\mathrm{mas}]$ \\
\hline Valinhos & $-2.00 \pm 12.00$ & $+34.00 \pm 18.00+38.00 \pm 19.00$ & +13.00 \\
FK5-VLBI & & & & \\
Martin 1998 & & & & \\
\hline ASPHO & $-8.60 \pm 0.93$ & $-0.65 \pm 0.94$ & $-2.70 \pm 0.67$ & +1.03 \\
(HIP-VLBI) & & & & \\
\hline Walter et al 1997 & $-26.90 \pm 0.50$ & $-12.60 \pm 0.60$ & $+23.00 \pm 0.50$ & - \\
\hline Li \& Jin 1996 & $-13.00 \pm 12.00+77.00 \pm 12.00$ & $+5.00 \pm 9.00$ & - \\
\hline
\end{tabular}

Da Tabela II, percebemos que os ângulos de rotação por nós determinados [12] são menores 
do que a dispersão de cada um deles (e também em outros sistemas). Isso é explicado pelo fato de termos um sistema local de referência. Os ângulos $A_{1}, A_{2}$ e $A_{3}$ são infinitesimais, o que está de acordo com a teoria física e geométrica de ângulos de rotação que diz que estes precisam ser infinitesimais em sistemas onde há uma relação direta entre os estados dinâmicos e as variáveis dinâmicas com o objetivo de garantir uma ligação entre a operação infinitesimal e o momento cinético dos corpos envolvidos, que no nosso caso são as estrelas.

\section{COMENTÁRIOS FINAIS}

No final do século XX, a Astrometria experimentou um grande avanço tecnológico, principalmente devido ao progresso da Física e Engenharia Eletrônica. Modernizou-se e entrou na chamada Era Espacial. Duas grandes missões espaciais têm trazido resultados primorosos na compreensão do universo: o Telescópio Espacial Hubble (HST), com objetivos astrofísicos e astrométricos, e a missão HIPPARCOS, com finalidade puramente astrométrica. Todavia, por exigir tecnologia avançada essas missões tornam-se caras e não-freqüentes, além de problemas técnicos, tendo em vista que não se consegue garantir a estabilidade nas plataformas de lançamento, o que irá repercutir no resultado final dos dados [6, 17]. O Astrolábio Fotoelétrico constitui-se numa ferramenta importante no que tange à confecção de catálogos estelares locais. Se compararmos os resultados obtidos com o ASPHO (sistema local de solo) com os da missão HIPPARCOS (sistema global no espaço), percebemos que o ASPHO nos propicia resultados satisfatórios [18].

Desta forma, no presente trabalho:

- conseguimos determinar, através do ASPHO, as coordenadas das estrelas dos nossos grupos, com boa precisão;

- durante o processo de conexão ópticorádio, as posições ópticas com o astrolábio em comparação às posições de- terminadas com técnicas rádio e com outras posições ópticas são muito boas;

- encontramos, ainda, ângulos de rotação coerentes com os determinados por [6] com técnica VLBI.

Apesar dos projetos espaciais audaciosos em astrometria óptica estarem em fase de desenvolvimento para esse século, como o SIM (Space Interferometry Mission), o FAME (Fullsky Astrometric Mapping Explorer) e o GAIA (Global Astrometric Interferometer for Astrophysics), a astrometria de solo continuará desempenhando seu papel. No caso do ASPHO, por exemplo, substituindo a fotomultiplicadora por uma câmara CCD haveria um ganho em magnitude. Estrelas de até magnitude 12 (limite superior) e pequenos planetas poderiam ser observados. Isso significa um ganho em precisão nos dados astrométricos de solo e obtenção dos elementos orbitais da Terra.

Dessa forma, podemos concluir, que o Astrolábio Fotoelétrico tem notável aplicação astronômica na contribuição ao estudo dos efeitos sistemáticos e correções ao sistema fundamental de referência (equador) por intermédio de um método de redução global. Juntamente com outros instrumentos ópticos, o ASPHO ainda desempenha um papel importante nesse novo momento de inserção da astronomia na era espacial, confeccionando catálogos estelares, conectando catálogos estelares locais à catálogos globais, corrigindo elementos principais do Sistema Solar, corrigindo constantes fundamentais para a astronomia ou, mais do que isso, propondo novos modelos, o que ratifica a posição de destaque que a Astrometria desempenha para o desenvolvimento da Astronomia, na busca do tão desejado Sistema Global Inercial de Referência.

\section{AGRADECIMENTOS}

$\mathrm{AAB}$ agradece ao $\mathrm{CNPq}$ pelas bolsas de pesquisa concedidas dentro do Programa PIBIC/CNPq de 1998 a 2001 e à UEFS. $\mathrm{AAB}$ agradece aos pesquisadores Rosa Bunchaft (UEFS), Hans Walter (Heidelberg) e 
Nicole Capitaine (Observatoire de Paris) pelas discussões e envio de artigos e livros.
[1] N. Capitaine, C.R.B. Guinot. Acad. Sci. Paris t-324, (II b) 725 (1997).

[2] J. Kovalevsky. Space Science Reviews 39, 1 (1984).

[3] J. Kovalevsky. Rep. Prog. Phys. 61, 77 (1998).

[4] K.J. Johnston et al.. Annu. Ver. Astron. Astrophys. 37, 971999.

[5] M.H. Soffel, Relativity in Astrometry, Celestial Mechanics and Geodesy. New York: SpringerVerlag (1989).

[6] Walter et al.. A \& AS 86, 357 (1990).

[7] T. Fukushima. Celestial Mechanics and Dynamical Astronomy 66, 107 (1997).

[8] H. Andrei, J.L. Penna. Boletim da SAB 12, (1) (1990)

[9] G. Billaud. IAUS 389B, 109 (1986).

[10] G. Billaud, R. Boche, M. Furia, C. Meyer, F. Mignard. A \& AS 67B, 68 (1987).

[11] A. Alves-Brito, Conexão entre os Sistemas de Referência Óptico e Rádio. Monografia (TAFC) - Departamento de Física da UEFS, Feira de Santana (2002).

[12] V.A.F. Martin, Contribuição ao estudo dos efeitos sistemáticos, sistemas de referência $e$ movimentos próprios . Tese (Doutorado) - Ins- tituto Astronômico e Geofísico da USP, São Paulo (1998).

[13] M.A.C. Perryman et al., The Hipparcos Mission. ESA-SP I-II, 1111 (1989).

[14] R. Boczko, Conceitos de Astronomia. São Paulo: Edgard Blucher (1984).

[15] P.C.R. Poppe, 20 anos de observações do Sol com os astrolábios dos sítios de Valinhos (OAM) e Calern (OCA) : correções ao sistema fundamental de referência (FK5) e aos elementos orbitais da Terra. Tese (Doutorado) - Instituto Astronômico e Geofísico da USP, São Paulo (1998).

[16] J. Lin, W. Jin. A\&A 118, 323 (1996).

[17] H. Walter, O.J. Sovers, Astrometry of Fundamental Catalogues. New York: SpringerVerlag (2000).

[18] S. Debarbat. Astrophysics and Space Science 177, 279 (1991).

[19] V.A.F. Martin, N.V. Leister, P.C.R. Poppe, A.A. Brito, L. Mattos. ADeLA Publications Series 1, 105 (2003).

[20] H.G. Walter, R. Hering, Chr. de Vegt. A \& AS 122, 529 (1997). 